\title{
Relation of Carotid Intima-Media Thickness and Plaque With Incident Cardiovascular Events in Women With Systemic Lupus Erythematosus
}

\author{
Amy H. Kao, MD, MPH, MS ${ }^{a},{ }^{\star}$, Apinya Lertratanakul, MD ${ }^{b}$, Jennifer R. Elliott, MD ${ }^{c}$, Abdus \\ Sattar, $\mathbf{P h D}^{\mathrm{d}}$, Linda Santelices, $\mathbf{M S}^{\mathrm{e}}$, Penny Shaw, $\mathbf{R N}^{\mathrm{c}}$, Mehret Birru, MD, $\mathbf{P h D}^{\mathrm{c}}$, Zheni \\ Avram, MD $^{c}$, Trina Thompson, $\operatorname{DrPH}^{f}$, Kim Sutton-Tyrrell, DrPH $^{f}$, Rosalind Ramsey- \\ Goldman, MD, DrPH ${ }^{b}$, and Susan Manzi, MD, MPH ${ }^{a, f}$ \\ aLupus Center of Excellence, Allegheny Health Network, Pittsburgh, Pennsylvania \\ bDivision of Rheumatology, Department of Medicine, Northwestern University, Feinberg School of \\ Medicine, Chicago, Illinois \\ cUniversity of Pittsburgh, Pittsburgh, Pennsylvania \\ ${ }^{d}$ Case Western Reserve University, Cleveland, Ohio \\ ${ }^{e}$ Allegheny-Singer Research Institute, Pittsburgh, Pennsylvania \\ fGraduate School of Public Health, University of Pittsburgh, Pittsburgh, Pennsylvania
}

\section{Abstract}

Patients with systemic lupus erythematosus (SLE) are at increased risk for cardiovascular (CV) disease. The aim of this study was to investigate the association between subclinical CV disease as measured by carotid intima-media thickness (IMT) and plaque using B-mode carotid ultrasound and incident CV events in a combined cohort of female patients with SLE. This was a prospective, 2-center observational study of 392 adult women with SLE and no previous CV events with a mean 8 years of follow-up. Incident CV events confirmed by clinicians were defined as angina, myocardial infarction, percutaneous transluminal coronary angioplasty, coronary artery bypass graft, fatal cardiac arrest, transient ischemic attack, and cerebrovascular accident. Incident hard $\mathrm{CV}$ events excluded angina and transient ischemic attack. The mean age was 43.5 years, and most patients were Caucasian (77.3\%). During follow-up, 38 patients had incident CV events, and 17 had incident hard $\mathrm{CV}$ events. Patients with incident hard $\mathrm{CV}$ events had higher mean carotid IMT ( 0.80 vs $0.64 \mathrm{~mm}, \mathrm{p}<0.01$ ) and presence of carotid plaque $(76.5 \%$ vs $30.4 \%, \mathrm{p}<0.01$ ) compared with those without incident hard CV events. Baseline carotid IMT and presence of plaque were predictive of any incident hard CV event (hazard ratio 1.35, 95\% confidence interval 1.12 to 1.64 , and hazard ratio $4.26,95 \%$ confidence interval 1.23 to 14.83 , respectively), independent of traditional CV risk factors and medication use. In conclusion, in women with SLE without previous $\mathrm{CV}$ events, carotid IMT and plaque are predictive of future $\mathrm{CV}$ events. This suggests that carotid ultrasound may provide an additional tool for CV risk stratification in patients with SLE.

Systemic lupus erythematosus (SLE), an autoimmune inflammatory disease primarily affecting women of child-bearing age, manifests with frequent disease flares that may

\footnotetext{
(c) 2013 Elsevier Inc. All rights reserved.

*Corresponding author: Tel: (412) 578-6815; fax: (412) 605-6669. akao@wpahs.org (A.H. Kao).

Disclosures

The authors have no conflicts of interest to disclose.
} 
ultimately lead to organ failure. Patients with SLE are at higher risk for cardiovascular (CV) disease (CVD) compared with women of similar ages. ${ }^{1-5}$ Young women aged 36 to 45 years with SLE were found to have a 50-fold greater risk for myocardial infarction compared with women of similar ages in the Framingham Offspring study. ${ }^{6}$ Cross-sectional and retrospective cohort studies have shown that hypertension, obesity, diabetes mellitus, smoking, hyperlipidemia, and a sedentary lifestyle play a role in accelerated atherosclerosis. ${ }^{2,3,7}$ However, the diagnosis of SLE remains a strong risk factor for CVD, even after controlling for traditional Framingham risk factors. ${ }^{8}$ The most important SLE factors contributing to premature CVD remain unknown. Noninvasive imaging techniques have been used to explore why SLE predisposes women to excess CVD burden. Increased rates of carotid plaque $(37.1 \% \text { vs } 15.2 \%)^{9}$ and coronary calcification $(30.7 \% \text { vs } 8.7 \%)^{10}$ have been reported in women with SLE compared with controls. Other studies have found higher than expected rates of subclinical atherosclerosis in women with SLE. ${ }^{9-11}$ To the best of our knowledge, noninvasive assessment of carotid intima-media thickness (IMT) and plaque have not been evaluated for prediction of risk for incident $\mathrm{CV}$ events in longitudinal studies of women with SLE. Noninvasive measures that can predict CV events would be invaluable. The purpose of this study was to determine whether baseline carotid IMT and plaque predict incident $\mathrm{CV}$ events in asymptomatic women with SLE.

\section{Methods}

A total of 392 women with SLE were enrolled in this study. We enrolled 289 women from the Pittsburgh Lupus Cardiovascular Study (1995 to 1998) as part of a longitudinal National Institutes of Health - funded study of CVD in patients with SLE. This cohort includes patients seen either at the University of Pittsburgh Medical Center inpatient and outpatient facilities or by rheumatologists in the Pittsburgh metropolitan area. Thus, the sample represents a community-based spectrum of mild to severe SLE with minimal tertiary care center referral bias. We also enrolled 185 women from the Study of Lupus Vascular and Bone Long-Term Endpoints (SOLVABLE) at Northwestern University, a National Institutes of Health-funded study. All subjects from the Pittsburgh Lupus Cardiovascular Study fulfilled the 1982 American College of Rheumatology revised criteria for the classification of SLE. ${ }^{12}$ All women from SOLVABLE fulfilled the 1982 or updated 1997 American College of Rheumatology classification criteria for $\mathrm{SLE}^{12,13}$ and were $\geq 18$ years of age. CVD history was defined as having any of the following: myocardial infarction, angina, cerebrovascular accident, transient ischemic attack, or coronary revascularization. Because the aim of this study was to investigate the predictive values of carotid IMT and plaque at baseline for incident CV events, we excluded subjects with SLE with histories of CVD as defined earlier. At baseline, each participant completed B-mode carotid ultrasound, an interview, a physical examination, and laboratory tests. In this study, the follow-up period for the Pittsburgh cohort began in July 1995 and ended in April 2008, and the follow-up period for the Chicago cohort began in December 2002 and ended in March 2012. Each participant provided written informed consent and authorization for release of medical information. The Pittsburgh Lupus Cardiovascular Study was approved by the University of Pittsburgh's Institutional Review Board, and SOLVABLE was approved by the Institutional Review Board of Northwestern University.

Study procedures were uniform between the sites; the study visit included anthropomorphic measurements (height, weight, and waist and hip circumferences), 2 consecutive blood pressure readings (with patients seated and at rest for $\geq 5$ minutes), and a blood draw after a required overnight fast. Blood samples were used to measure total cholesterol, triglycerides, and high-density lipoprotein cholesterol at the Lipid Laboratory in the University of Pittsburgh Graduate School of Public Health, which has been Clinical Laboratory Improvement Amendments certified by the Centers for Disease Control and Prevention. 
Low-density lipoprotein cholesterol was calculated from measured total cholesterol, highdensity lipoprotein, and triglycerides (using the Friedewald equation). ${ }^{14}$ Hypertension was defined by physician diagnosis, measured mean blood pressure $\geq 140 / 90 \mathrm{~mm} \mathrm{Hg}$, or antihypertensive medication use. Information was also collected on family history of CVD (first-degree relative having a minor stroke before 60 years of age), cigarette smoking (current, past, or never), childbearing history, and menopausal status.

Information was recorded on corticosteroid treatment (current, ever, and dosage) and current use of hydroxychloroquine, immunosuppressant agents, nonsteroidal anti-inflammatory drugs, antihypertensive drugs, hormonal therapy, and lipid-lowering medications. SLE disease activity was measured by the Systemic Lupus Activity Measure. ${ }^{15}$ Cumulative damage from SLE and/or its treatment was measured using the Systemic Lupus International Collaborating Clinics damage index. ${ }^{16}$ Antiphospholipid antibodies, including anticardiolipin immunoglobulin G/immunoglobulin $\mathrm{M}$ and lupus anticoagulant (i.e., mixed partial thromboplastin time and dilute Russell viper venom time), were measured. An enzyme-linked immunosorbent assay was used for the determination of high-sensitivity Creactive protein. All specialized laboratory tests (e.g., lipids, inflammatory markers, antiphospholipid antibodies) for the 2 sites were performed at the same laboratory.

The sonographers from the 2 study sites were trained at the University of Pittsburgh Ultrasound Research Laboratory. Carotid ultrasound was performed at the University of Pittsburgh Ultrasound Research Laboratory and at the Northwestern University, as previously described. All carotid ultrasound studies were read at the University of Pittsburgh Ultrasound Research Laboratory. The common carotid artery, carotid bulb, and the first 1.5 $\mathrm{cm}$ of the proximal internal carotid artery and external carotid artery were digitized bilaterally using a 5-MHz linear-array transducer on a Toshiba 140 (Toshiba, Tustin, California) ultrasound machine. IMT readings were performed across 1-cm segments for baseline and follow-up exams by a consistent reader using automated edge-detection software (AMS, Stockholm, Sweden). Readings were blinded and spaced $\geq 1$ month apart. Values from these 8 measures were averaged to obtain the mean average IMT. The intraclass correlation coefficient for carotid IMT was $\searrow 0.90$ between sonographers and 0.87 between readers.

Plaque was defined as a focal projection within the intima-media layer that was $250 \%$ greater than adjacent IMT. The number of plaques was totaled bilaterally. The plaque index was calculated by summing the number of plaques and plaque grades present in the bilateral common carotid artery, bulb, and proximal ICA, which provided an estimate of overall plaque burden.

$\mathrm{CV}$ events were defined as angina, myocardial infarction, percutaneous transluminal coronary angioplasty, coronary artery bypass grafting, fatal cardiac arrest, transient ischemic attack, and/or cerebrovascular accident. CV events were confirmed by (1) patient report with physician confirmation, (2) chart review (by JRE, ZA, and RR-G), or (3) National Death Index causes of death. The outcome variables were (1) time to any incident CV event, which included all CV events, and (2) time to any incident hard CV event, which excluded angina and transient ischemic attack. Of the 289 women with SLE enrolled at baseline in Pittsburgh, 65 with previous CV events were excluded from this study and 4 were lost to follow-up; of the 185 women with SLE enrolled at baseline in Chicago, 12 with previous $\mathrm{CV}$ events were excluded and 1 did not have baseline carotid measures. Therefore, the present study sample was composed of the remaining 392 (220 from Pittsburgh and 172 from Chicago) women with SLE and without CV events. 
The 10-year absolute risk score for hard CV events was calculated using gender-specific regression equations described by the Framingham Heart Study, which included age, smoking, diabetes, systolic blood pressure, antihypertensive medication use, and total and high-density lipoprotein cholesterol. ${ }^{17}$ Low, intermediate, and high risk in that study were defined as $0 \%$ to $<6 \%, 6 \%$ to $20 \%$, and $>20 \%$, respectively. Because our mean observed follow-up at the time of analysis was 8 years, we redefined the low-, intermediate-, and high-risk intervals for 8 -year risk for hard CV events to be $0 \%$ to $<4 \%, 4 \%$ to $14 \%$, and $>14 \%$ on the basis of the proportionality assumption of incidence rate. ${ }^{18}$

Descriptive statistics are reported as mean $\pm \mathrm{SD}$ or as median (interquartile range) for continuous variables on the basis of distribution and as percentages for categorical variables. The means or medians of continuous variables were compared using either Student's $t$ tests or Wilcoxon's rank-sum tests, depending on the distribution of the data. Categorical variables were analyzed using Fisher's exact or chi-square tests. Age-adjusted and multivariate Cox proportional-hazards models were used to identify predictors of time to any incident $\mathrm{CV}$ event and time to any incident hard CV event. Carotid IMT and plaque were treated as 2 separate predictors in the regression models. The test of proportional assumptions was also performed. Kaplan-Meier survival analysis and log-rank and Wilcoxon's tests for equality were performed for carotid plaque. All tests were 2 sided, with a significance level of 0.05. Analyses were performed using Stata SE version 11.0 for Windows (StataCorp, College Station, Texas).

\section{Results}

The 392 female subjects with SLE had a mean age of 43.5 years and were predominantly Caucasian (77.3\%). The 2 study sites differed by ethnicity, level of education, and duration of follow-up (Table 1). The mean SLE disease duration was 10.7 years, and the mean Systemic Lupus International Collaborating Clinics damage score was 1.27 (Table 2). The overall mean duration of follow-up was 7.9 years. A total of 65 new CV events occurred during follow-up: 22 episodes of angina, 10 myocardial infarctions, 10 percutaneous transluminal coronary angioplasty procedures, 5 coronary artery bypass graft procedures, 3 cardiac arrests, 7 transient ischemic attacks, and 8 cerebrovascular accidents. A total of 38 patients had incident $\mathrm{CV}$ events and 17 patients had incident hard CV events. The incidence rates per 1,000 person-years for any CV events and any hard CV events were 0.04 and 0.02 , respectively. The SLE-specific factors, including SLE duration, disease activity, damage, and clinical manifestations by American College of Rheumatology criteria were not significantly different between those with and without any incident hard CV events (Table 2). However, compared with patients without incident hard CV events, those with any incident hard CV events were significantly older, reported longer durations of glucocorticoid use, had higher total cholesterol levels, had higher systolic blood pressures, and were more likely to be taking lipid-lowering drugs at baseline. Furthermore, women with SLE and with any incident hard CV events also had significantly higher mean carotid IMT at baseline ( $0.80 \mathrm{vs} 0.64 \mathrm{~mm})$ and were more likely to have carotid plaque at baseline ( $76.5 \%$ vs $30.4 \%)$ compared with those without any incident hard CV events (all $\mathrm{p}$ values $<0.01$ ).

Longer SLE disease duration and duration of glucocorticoid use at baseline were significantly associated with any incident $\mathrm{CV}$ event in age-adjusted Cox regression models (Table 3). Carotid IMT (hazard ratio [HR] 1.14, 95\% confidence interval [CI] 1.00 to 1.31, p $=0.05)$ and plaque at baseline (HR 1.83, 95\% CI 0.91 to $3.66, \mathrm{p}=0.09)$ were borderline predictors of any incident CV events in age-adjusted Cox regression models. However, carotid IMT and carotid plaque at baseline were significant predictors of incident hard CV events (Tables 3 and 4). Carotid plaque at baseline was associated with a nearly 5 -fold increased risk for any incident hard CV event (HR 4.67, 95\% CI 1.41 to $15.53, \mathrm{p}=0.01$ ), 
whereas carotid IMT was associated with an HR of 1.35 (95\% CI 1.15 to $1.59, \mathrm{p}<0.01$ ) in the separate multivariate Cox regression models. The significant association between carotid measures and incident hard CV events was independent of other significant predictors, including Framingham risk score, lipid-lowering medication use, and longer duration of glucocorticoid use. The addition of study site location, smoking history, menopausal status, antiphospholipid antibodies, Systemic Lupus International Collaborating Clinics damage score, hormonal therapy, or immunosuppressive therapy did not attenuate the significant association in the multivariate regression models. The proportionality assumption was met. Figure 1 illustrates the Kaplan-Meier estimates for incident hard CV events stratified by patients with SLE with and without carotid plaque. Log-rank tests and Wilcoxon's tests for equality of survival showed significant differences between the 2 groups ( $p<0.01$ for both). Figure 2 illustrates that patients with SLE and without carotid plaque at baseline appeared to have lower risk for any incident hard CV event, regardless of Framingham risk category, compared with those with carotid plaque at baseline.

\section{Discussion}

In this cohort of women with SLE who were free of CV events at study entry, carotid IMT and the presence of carotid plaque at baseline were significantly associated with incident hard $\mathrm{CV}$ events during a mean follow-up period of 7.9 years. The presence of carotid plaque at baseline was associated with a greater than fourfold increased risk for any hard CV events. Kaplan-Meier estimates indicated that the presence of plaque was related to a higher rate of any $\mathrm{CV}$ event compared with the absence of plaque. These results suggest that indicators of preclinical CVD are associated with worse CV outcomes over time.

Several population-based studies have shown significant associations between incident CV events and carotid IMT. ${ }^{19-22}$ In the Framingham Offspring cohort, the maximal and mean common carotid IMT measurements were predictive of CV outcomes, whereas the presence of plaque significantly improved the prediction of incident $\mathrm{CV}$ events and added predictive value to the Framingham risk score. Similar to these population-based studies, we also found the predictive value of carotid IMT and plaque with incident CV events to be independent of known modifiable CV risk factors in women with SLE, such as hypertension and hyperlipidemia requiring lipid-lowering medication. Similar to non-SLE studies, we found a significant association between longer duration of glucocorticoid use at baseline and incident hard CV events. ${ }^{23,24}$ This association may be explained by the metabolic disorder induced by glucocorticoids and the need for continued use of glucocorticoids to treat SLE. ${ }^{25-27}$ Glucocorticoids exert significant immunosuppressant and anti-inflammatory effects that are important and often necessary in the management of SLE but are not without side effects, including osteonecrosis and osteoporosis. Many studies have also found associations between glucocorticoid use and higher rates of CVD.

Although an increased risk for CVD is present in patients with SLE, there is evidence suggesting glucocorticoids themselves may be a contributing factor. Longer duration of prednisone use and higher cumulative prednisone dose have been associated with presence of carotid plaque, ${ }^{11}$ and years of steroid use is associated with progression of IMT in women with SLE. ${ }^{28}$ However, more active SLE may suggest the need for more or longer glucocorticoid use, and it is unclear if there is a direct relation between glucocorticoid use and CVD or if it is a marker of cumulative disease activity. ${ }^{29}$

Our study had some limitations and strengths. Our combined cohort consists of only female patients with SLE, predominantly Caucasian, who had mild SLE disease and damage on the basis of their Systemic Lupus Activity Measure and Systemic Lupus International Collaborating Clinics scores, respectively. Therefore, our findings may not be generalized to 
male patients with SLE, non-Caucasians, or those with more severe SLE. Because of the limited number of $\mathrm{CV}$ events observed during the study period, the $\mathrm{CI}$ for the association between carotid plaque and hard CV events was wide. Instead of measuring only the common carotid artery, as was done in many studies, we used all segments of carotid arteries in the measurement of carotid IMT. Although our combined cohort was from 2 different study sites, the carotid ultrasonographers were trained by the same site, and all carotid ultrasound studies were also read at the same site.

Our findings suggest that these noninvasive carotid measures may have independent value in the estimation of future CV outcomes in CV event-free women with SLE. In women with SLE, who are already known to have greater CV risks compared with patients without SLE, the presence of carotid plaque increases the risk for incident $\mathrm{CV}$ events. Therefore, carotid measures, especially the presence of carotid plaque, in CV event-free women with SLE should alert clinicians to treat modifiable CV risk factors more aggressively. Awareness of the additional $\mathrm{CV}$ risk posed by the presence of carotid plaque may also encourage these women with SLE to adhere to prescribed medical management for CVD prevention.

\section{Acknowledgments}

We thank the study participants and the funding agencies. We thank Dr. William Pearce, Department of Vascular Surgery, Northwestern University Feinberg School of Medicine, and Dr. David D. McPherson, Division of Cardiovascular Medicine, University of Texas Houston, for providing oversight for the carotid ultrasound examinations for SOLVABLE. We also thank Michael Anderson for his editorial assistance.

This study was funded by the Arthritis Foundation, Lupus Foundation of America, Western Pennsylvania Chapter, grant-in-aid from the American Heart Association, grant R01 AR046588-01 and P60 AR030692 from the National Institutes of Health, M01-RR000056 and M01-RR000048 from the NIH/NCRR/GCRC, and P60 AR044811-01 from the NIH/MAC. Additional author support: AHK (ACR REF Physician Scientist Development Award; NIH K23 AR051044), AL (ACR REF/Amgen Rheumatology Fellowship Training Award; The Driskill Foundation), RR-G (NIH/NCATS UL 1 RR025741; NIH K24 AR002138), SM (NIH K24 AR002213-01).

\section{References}

1. Urowitz M, Bookman A, Koehler B, Smythe H, Ogryzlo M. The bimodal mortality pattern of systemic lupus erythematosus. Am J Med. 1976; 60:221-225. [PubMed: 1251849]

2. Petri M, Perez-Gutthann S, Spence D, Hochberg M. Risk factors for coronary artery disease in patients with systemic lupus erythematosus. Am J Med. 1992; 93:513-519. [PubMed: 1442853]

3. Gladman D, Urowitz M. Morbidity in systemic lupus erythematosus. J Rheumatol. 1987; 14:223226.

4. Ward M. Premature morbidity from cardiovascular and cerebrovascular diseases in women with systemic lupus erythematosus. Arthritis Rheum. 1999; 42:338-346. [PubMed: 10025929]

5. Ward M. Cardiovascular and cerebrovascular morbidity and mortality among women with end-stage renal disease attributable to lupus nephritis. Am J Kidney Dis. 2000; 36:516-525. [PubMed: 10977783]

6. Manzi S, Meilahn E, Rairie J, Conte C, Medsger T, Jansen-McWilliams L, D’Agostino R, Kuller L. Age-specific incidence rates of myocardial infarction and angina in women with systemic lupus erythematosus: comparison with the Framingham study. Am J Epidemiol. 1997; 145:408-415. [PubMed: 9048514]

7. Chung CP, Avalos I, Oeser A, Gebretsadik T, Shintani A, Raggi P, Michael Stein C. High prevalence of the metabolic syndrome in patients with systemic lupus erythematosus: association with disease characteristics and cardiovascular risk factors. Ann Rheum Dis. 2007; 66:208-214. [PubMed: 16901956]

8. Esdaile J, Abrahamowicz M, Grodzicky T, Li Y, Panaritis C, du Berger R, Cote R, Grover S, Fortin P, Clarke A, Senecal J. Traditional Framingham risk factors fail to fully account for accelerated atherosclerosis in systemic lupus erythematosus. Arthritis Rheum. 2001; 44:2331-2337. [PubMed: 11665973] 
9. Roman M, Shanker B, Davis A, Lockshin M, Sammaritano L, Simantov R, Crow M, Schwartz J, Paget S, Devereux R, Salmon J. Prevalence and correlates of accelerated atherosclerosis in systemic lupus erythematosus. N Engl J Med. 2003; 349:2399-2406. [PubMed: 14681505]

10. Asanuma Y, Oeser A, Shintai A, Turner E, Olsen N, Fazio S, Linton M, Raggi P, Stein C. Premature coronary-artery atherosclerosis in systemic lupus erythematosus. N Engl J Med. 2003; 349:2407-2415. [PubMed: 14681506]

11. Manzi S, Selzer F, Sutton-Tyrrell K, Fitzgerald S, Rairie J, Tracy R, Kuller L. Prevalence and risk factors of carotid plaque in women with systemic lupus erythematosus. Arthritis Rheum. 1999; 42:51-60. [PubMed: 9920014]

12. Tan E, Cohen A, Fries J. The 1982 revised criteria for the classification of systemic lupus erythematosus. Arthritis Rheum. 1982; 25:1271-1277. [PubMed: 7138600]

13. Hochberg M. Updating the American College of Rheumatology revised criteria for the classification of systemic lupus erythematosus. Arthritis Rheum. 1997; 40:1725. [PubMed: 9324032]

14. Friedewald W, Levy R, Fredrickson D. Estimation of the concentration of low-density lipoprotein cholesterol in plasma without use of the preparative ultracentrifuge. Clin Chem. 1972; 18:499 502. [PubMed: 4337382]

15. Liang M, Socher S, Larson M, Schur P. Reliability and validity of six systems for the clinical assessment of disease activity in systemic lupus erythematosus. Arthritis Rheum. 1989; 32:11071118. [PubMed: 2775320]

16. Gladman D, Ginzler E, Goldsmith C, Fortin P, Liang M, Urowitz M, Bacon P, Bombardieri S, Hanly J, Hay E, Isenberg D, Jones J, Kalunian K, Maddison P, Nived O, Petri M, Richter M, Sanchez-Guerrero J, Snaith M, Sturfelt G, Symmons D, Zoma A. The development and initial validation of the Systemic Lupus International Collaborating Clinics/American College of Rheumatology damage index for systemic lupus erythematosus. Arthritis Rheum. 1996; 39:363369. [PubMed: 8607884]

17. D’Agostino RB, Vasan RS, Pencina MJ, Wolf PA, Cobain M, Massaro JM, Kannel WB. General cardiovascular risk profile for use in primary care: the Framingham Heart Study. Circulation. 2008; 117:743-753. [PubMed: 18212285]

18. Pencina MJ, Steyerberg E, D’Agostino RB. Extensions of net reclassification improvement calculations to measure usefulness of new biomarkers. Stat Med. 2011; 30:11-21. [PubMed: 21204120]

19. Chambless LE, Heiss G, Folsom AR, Rosamond W, Szklo M, Sharrett AR, Clegg LX. Association of coronary heart disease incidence with carotid arterial wall thickness and major risk factors: the Atherosclerosis Risk in Communities (ARIC) study 1987-1993. Am J Epidemiol. 1997; 146:483494. [PubMed: 9290509]

20. Lorenz MW, von Kegler S, Steinmetz H, Markus HS, Sitzer M. Carotid intima-media thickening indicates a higher vascular risk across a wide age range. Stroke. 2006; 37:87-92. [PubMed: 16339465]

21. Rosvall M, Janzon L, Berglund G, EngstrOM G, Hedblad B. Incident coronary events and case fatality in relation to common carotid intima-media thickness. J Intern Med. 2005; 257:430-437. [PubMed: 15836659]

22. Polak JF, Pencina MJ, Pencina KM, O’Donnell CJ, Wolf PA, D’ Agostino RB. Carotid-wall intima-media thickness and cardiovascular events. N Engl J Med. 2011; 365:213-221. [PubMed: 21774709]

23. Wei L, MacDonald TM, Walker BR. Taking glucocorticoids by prescription is associated with subsequent cardiovascular disease. Ann Intern Med. 2004; 141:764-770. [PubMed: 15545676]

24. Laurence F, Irene P, Irwin N. Risk of cardiovascular events in people prescribed glucocorticoids with iatrogenic Cushing's syndrome: cohort study. BMJ. 2012; 345:e4928. [PubMed: 22846415]

25. Parker B, Bruce I. The metabolic syndrome in systemic lupus erythematosus. Rheum Dis Clin North Am. 2010; 36:81-97. [PubMed: 20202592]

26. Parker B, Ahmad Y, Shelmerdine J, Edlin H, Yates A, Teh L-S, Bruce I. An analysis of the metabolic syndrome phenotype in systemic lupus erythematosus. Lupus. 2011; 20:1459-1465. [PubMed: 21893561] 
27. Parker B, Urowitz MB, Gladman DD, Lunt M, Ibañez D, Hanly JG, Gordon C, Bae S-C, SanchezGuerrero J, Romero-Diaz J, Wallace DJ, Clarke AE, Ginzler EM, Merrill JT, Isenberg DA, Rahman A, Petri M, Fortin PR, Steinsson K, Dooley MA, Khamashta MA, Alarcón GS, Fessler BJ, Ramsey-Goldman R, Manzi S, Zoma AA, Sturfelt GK, Nived O, Aranow C, Mackay M, Ramos-Casals M, van Vol-lenhoven RF, Kalunian KC, Ruiz-Irastorza G, Lim S, Kamen DL, Peschken CA, Inanc M, Farewell V, Bruce IN. Clinical associations of the metabolic syndrome in systemic lupus erythematosus: data from an international inception cohort. Ann Rheum Dis. 2012 Sep 22. [Epub ahead of print].

28. Thompson T, Sutton-Tyrrell K, Wildman RP, Kao A, Fitzgerald SG, Shook B, Tracy RP, Kuller LH, Brockwell S, Manzi S. Progression of carotid intima-media thickness and plaque in women with systemic lupus erythematosus. Arthritis Rheum. 2008; 58:835-842. [PubMed: 18311797]

29. Skamra C, Ramsey-Goldman R. Management of cardiovascular complications in systemic lupus erythematosus. Int J Clin Rheumatol. 2010; 5:75-100. 


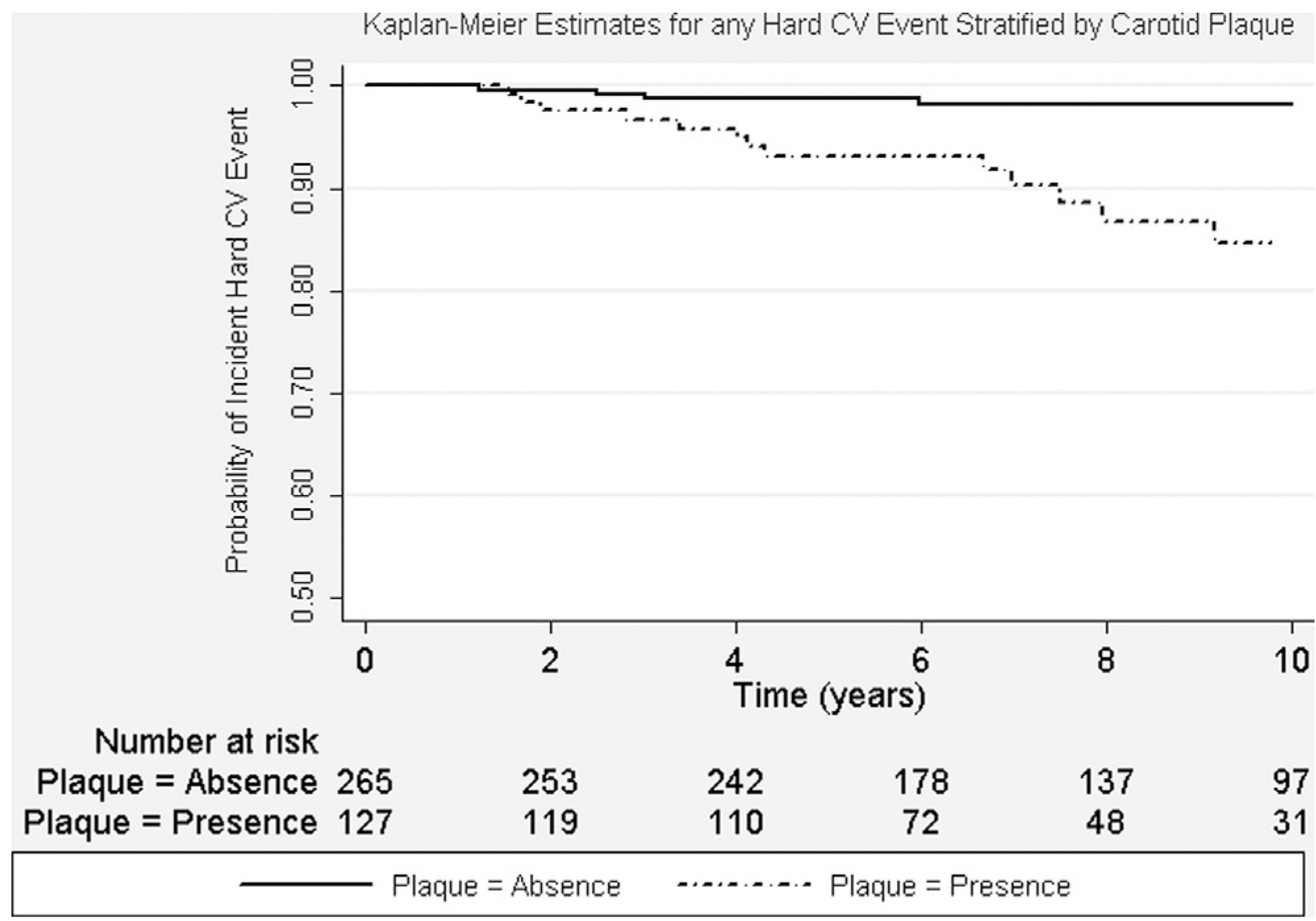

Figure 1.

Kaplan-Meier estimates for time to any incident hard CV event by carotid plaque. Women with SLE with carotid plaque (solid line) were more likely to have any incident hard CV events compared with those without carotid plaque (black line with a dot) at follow-up ( $\mathrm{p}$ $<0.01$ by log-rank and Wilcoxon's tests). 
A

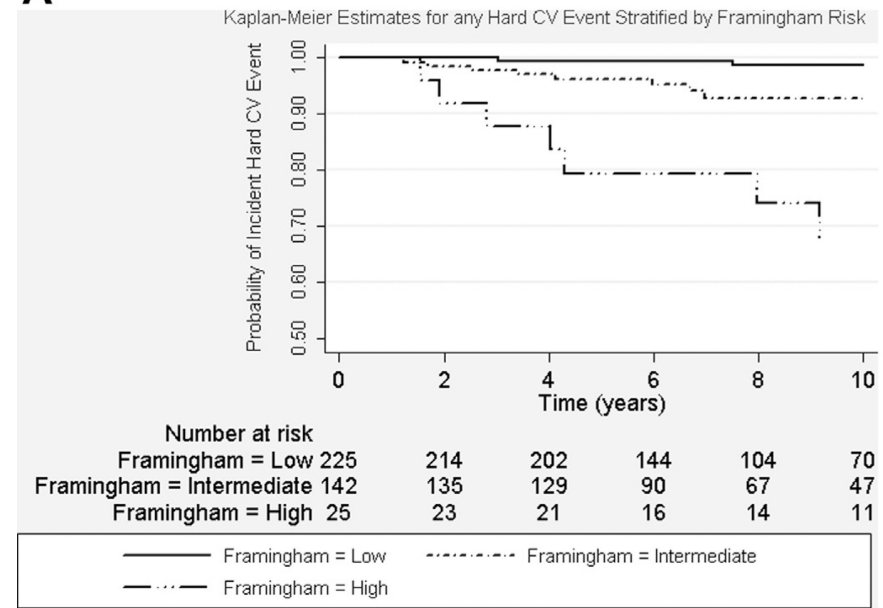

B

Kaplan-Meier Estimates for any Hard CV Event Stratified by Framingham Risk with Carotid Plaque

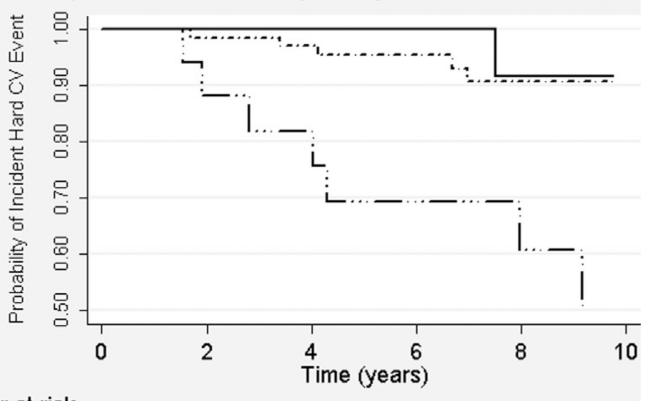

Number at risk

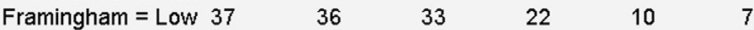

$\begin{array}{lllllll}\text { Framingham }=\text { High } & 17 & 15 & 13 & 8 & 7 & 5\end{array}$

Framingham = High $17 \quad 15 \quad 13 \quad 8$

- ... - Framingham $=$ High

C

Kaplan-Meier Estimates for any Hard CV Event Stratified by Framingham Risk without Carotid Plaque

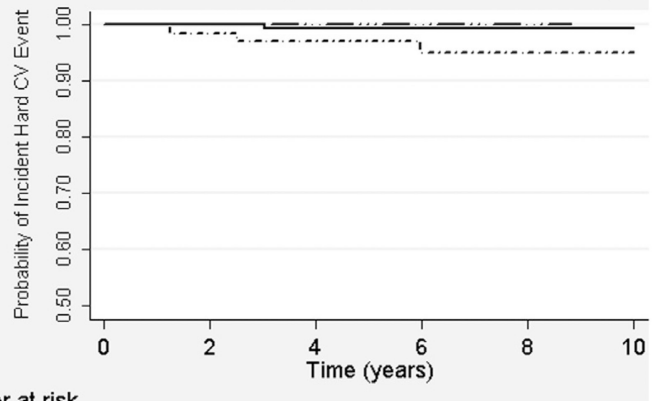

Number at risk

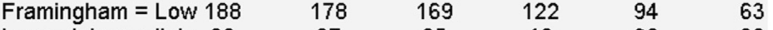

Framingham $=$ High 8

- Framingham $=$ Low

......... Framingham $=$ Intermediate

- $\cdots-$ Framingham $=$ High

Figure 2.

Kaplan-Meier estimates for time to any incident hard CV event by Framingham risk groups: (A) all women with SLE (p <0.01 by log-rank and Wilcoxon's tests); $(B)$ with carotid plaque ( $\mathrm{p}<0.01$ by log-rank and Wilcoxon's tests); and $(C)$ without carotid plaque $(\mathrm{p}=0.11$ by log-rank test and $\mathrm{p}=0.09$ by Wilcoxon's test). 


\section{Table 1}

Demographic characteristics of women with systemic lupus erythematosus by study site

\begin{tabular}{lcccr}
\hline Variable & Pittsburgh & & Chicago & p Value \\
$(\mathbf{n = 2 2 0})$ & & $(\mathbf{n = 1 7 2 )}$ & \\
\hline Age (yrs) & $51.4 \pm 11.4$ & $43.1 \pm 10.1$ & 0.15 \\
Caucasian & $198(90 \%)$ & $105(61.1 \%)$ & $<0.01$ \\
African-American & $9(8.6 \%)$ & $48(27.9)$ & \\
Others & $3(1.4 \%)$ & $19(11.0 \%)$ & \\
Education (yrs) & $14(12-16)$ & $16(12-18)$ & $<0.01$ \\
Follow-up (yrs) & $10 \pm 2.1$ & $5.2 \pm 1.6$ & $<0.01$ \\
\hline
\end{tabular}

Data are expressed as mean $\pm \mathrm{SD}$, number (percentage), or median (interquartile range). 
Table 2

Baseline characteristics of women with systemic lupus erythematosus

\begin{tabular}{|c|c|c|c|c|}
\hline \multirow[t]{2}{*}{ Variable } & \multirow[t]{2}{*}{ All Subjects $(n=392)$} & \multicolumn{2}{|c|}{ Hard CV Events } & \multirow[t]{2}{*}{ p Value } \\
\hline & & Yes $(n=17)$ & No $(n=375)$ & \\
\hline Age (yrs) & $43.5 \pm 10.4$ & $51.4 \pm 11.4$ & $43.1 \pm 10.1$ & $<0.01$ \\
\hline Caucasian & $303(77.3 \%)$ & $15(88 \%)$ & $288(76.8 \%)$ & 0.91 \\
\hline African-American & $67(17.1 \%)$ & $2(12 \%)$ & $65(17.3 \%)$ & \\
\hline Others & $22(5.6 \%)$ & 0 & $22(5.9 \%)$ & \\
\hline Education (yrs) & $15(12-17)$ & $13(12-14)$ & $15(12-17)$ & 0.01 \\
\hline Family history of CVD & $176 / 380(46 \%)$ & $8 / 14(57 \%)$ & $168 / 366(46 \%)$ & 0.43 \\
\hline Postmenopausal & $144(36.7 \%)$ & $11(65 \%)$ & $133(35.5 \%)$ & 0.01 \\
\hline \multicolumn{5}{|l|}{ Smokers } \\
\hline Ever & $160(40.8 \%)$ & $10(59 \%)$ & $150(40 \%)$ & 0.12 \\
\hline Current & $50(12.8 \%)$ & $3(18 \%)$ & $47(12.5 \%)$ & 0.47 \\
\hline Diabetes mellitus ${ }^{*}$ & $17(4.3 \%)$ & $1(6 \%)$ & $16(4.3 \%)$ & 0.54 \\
\hline Hypertension ${ }^{*}$ & $142(36.2 \%)$ & $14(82 \%)$ & $128(34.1 \%)$ & $<0.01$ \\
\hline Systolic blood pressure (mm Hg) & $118.4 \pm 16.8$ & $134.5 \pm 19.3$ & $117.7 \pm 16.3$ & $<0.01$ \\
\hline Diastolic blood pressure $(\mathrm{mm} \mathrm{Hg})$ & $75.8 \pm 10.2$ & $78.8 \pm 11.7$ & $75.7 \pm 10.1$ & 0.28 \\
\hline Total cholesterol & & & & 0.03 \\
\hline $\mathrm{mmol} / \mathrm{L}$ & $4.9 \pm 1.1$ & $5.6 \pm 1.2$ & $4.9 \pm 1.1$ & \\
\hline $\mathrm{mg} / \mathrm{dl}$ & $190.2 \pm 41.4$ & $214.3 \pm 47.3$ & $189.1 \pm 40.8$ & \\
\hline \multicolumn{5}{|l|}{ High-density lipoprotein cholesterol } \\
\hline $\mathrm{mmol} / \mathrm{L}$ & $1.5 \pm 0.4$ & $1.4 \pm 0.3$ & $1.5 \pm 0.4$ & 0.97 \\
\hline $\mathrm{mg} / \mathrm{dl}$ & $56.0 \pm 15.7$ & $55.2 \pm 11.4$ & $56.1 \pm 15.9$ & \\
\hline \multicolumn{5}{|l|}{ Low-density lipoprotein cholesterol } \\
\hline $\mathrm{mmol} / \mathrm{L}$ & $2.8 \pm 0.9$ & $3.3 \pm 1.1$ & $2.8 \pm 0.8$ & 0.12 \\
\hline $\mathrm{mg} / \mathrm{dl}$ & $109.0 \pm 32.9$ & $126.0 \pm 41.1$ & $108.3 \pm 32.4$ & \\
\hline \multicolumn{5}{|l|}{ Triglycerides } \\
\hline $\mathrm{mmol} / \mathrm{L}$ & $1.39 \pm 0.9$ & $1.7 \pm 0.9$ & $1.4 \pm 0.9$ & 0.07 \\
\hline $\mathrm{mg} / \mathrm{dl}$ & $123.3 \pm 80.9$ & $146.2 \pm 82.8$ & $122.3 \pm 80.8$ & \\
\hline \multicolumn{5}{|l|}{ Glucose } \\
\hline $\mathrm{mmol} / \mathrm{L}$ & $5.3 \pm 1.01$ & $5.1 \pm 0.7$ & $5.3 \pm 1.0$ & 0.42 \\
\hline $\mathrm{mg} / \mathrm{dl}$ & $94.8 \pm 18.3$ & $92.5 \pm 12.8$ & $94.9 \pm 18.5$ & \\
\hline Body mass index $\left(\mathrm{kg} / \mathrm{m}^{2}\right)$ & $27.2 \pm 6.8$ & $28.3 \pm 5.5$ & $27.1 \pm 6.9$ & 0.25 \\
\hline Waist circumference $(\mathrm{cm})$ & $87.5 \pm 16.6$ & $92.5 \pm 12.8$ & $94.9 \pm 18.5$ & 0.42 \\
\hline Serum creatinine (mg/dl) & $0.99 \pm 1.1$ & $0.88 \pm 0.2$ & $0.99 \pm 1.2$ & 0.26 \\
\hline SLE duration (yrs) & $10.7 \pm 7.9$ & $13.7 \pm 10.7$ & $10.6 \pm 7.7$ & 0.35 \\
\hline Systemic Lupus Activity Measure score & $6.5 \pm 3.6$ & $6.3 \pm 4.0$ & $6.5 \pm 3.6$ & 0.63 \\
\hline Systemic Lupus International Collaborating Clinics damage score & $1.3 \pm 1.61$ & $1.7 \pm 1.7$ & $1.3 \pm 1.6$ & 0.17 \\
\hline High-sensitivity C-reactive protein (mg/L) & $1.6(0.6-4.1)$ & $3.8(1-7.8)$ & $1.6(0.6-3.9)$ & 0.09 \\
\hline Complement C3 (g/L) & $94.5 \pm 25.9$ & $95.1 \pm 32.4$ & $94.5 \pm 25.6$ & 0.74 \\
\hline Complement C4 (g/L) & $20.3 \pm 8.3$ & $21.7 \pm 9.5$ & $20.3 \pm 8.2$ & 0.75 \\
\hline
\end{tabular}




\begin{tabular}{|c|c|c|c|c|}
\hline \multirow[t]{2}{*}{ Variable } & \multirow[t]{2}{*}{ All Subjects $(n=392)$} & \multicolumn{2}{|c|}{ Hard CV Events } & \multirow[t]{2}{*}{ p Value } \\
\hline & & Yes $(n=17)$ & No $(n=375)$ & \\
\hline Antinuclear antibody & $380(97 \%)$ & $17(100 \%)$ & $363(97 \%)$ & 1.0 \\
\hline Antiphospholipid antibodies & $128 / 386(33 \%)$ & $5(29 \%)$ & $123 / 369(33 \%)$ & 0.74 \\
\hline Lupus anticoagulant & $52 / 386(14 \%)$ & $3(18 \%)$ & $49 / 369(13 \%)$ & 0.49 \\
\hline Anticardiolipin antibodies & $79 / 385(21 \%)$ & $4(24 \%)$ & $75 / 368(20 \%)$ & 0.75 \\
\hline Anti-double-stranded deoxyribonucleic acid antibody & $125 / 390(32 \%)$ & $6(35 \%)$ & $119 / 373(32 \%)$ & 0.79 \\
\hline \multicolumn{5}{|l|}{ Medications at baseline } \\
\hline Length of glucocorticoid use (yrs) & $0.75(0.11-4)$ & $7(1-17)$ & $0.6(0.1-4)$ & 0.01 \\
\hline Glucocorticoid & $165(42 \%)$ & $10(59 \%)$ & $155(41 \%)$ & 0.15 \\
\hline Aspirin & $41 / 319(13 \%)$ & $0 / 5(0 \%)$ & $41 / 314(13 \%)$ & 0.39 \\
\hline Warfarin & $25(6 \%)$ & $2(12 \%)$ & $23(6 \%)$ & 0.30 \\
\hline Lipid-lowering drugs & $18 / 388(5 \%)$ & $3(18 \%)$ & $15 / 371(4 \%)$ & 0.04 \\
\hline Hydroxychloroquine & $228(58 \%)$ & $7(41 \%)$ & $221(59 \%)$ & 0.21 \\
\hline Nonsteroidal anti-inflammatory drugs & $137(35 \%)$ & $8(47 \%)$ & $129(34 \%)$ & 0.31 \\
\hline Immunosuppressant agents ${ }^{\dagger}$ & $82(21 \%)$ & $2(12 \%)$ & $80(21 \%)$ & 0.54 \\
\hline Hormonal therapy & $57(15 \%)$ & $5(29 \%)$ & $52(14 \%)$ & 0.08 \\
\hline \multicolumn{5}{|c|}{ SLE manifestations by the American College of Rheumatology criteria } \\
\hline Skin (malar/discoid rash) & $246(63 \%)$ & $10(59 \%)$ & $196(52 \%)$ & 0.78 \\
\hline Photosensitivity & $268(68 \%)$ & $12(71 \%)$ & $256(68 \%)$ & 1.0 \\
\hline Oral ulcer & $229(58 \%)$ & $9(53 \%)$ & $220(59 \%)$ & 0.83 \\
\hline Arthritis & $360(92 \%)$ & $17(100 \%)$ & $343(92 \%)$ & 0.38 \\
\hline Serositis & $167(43 \%)$ & $8(47 \%)$ & $159(42 \%)$ & 0.80 \\
\hline Renal & $119(30 \%)$ & $6(35 \%)$ & $113(30 \%)$ & 0.60 \\
\hline Central nervous system (seizure/psychosis) & $34(9 \%)$ & $1(6 \%)$ & $33(9 \%)$ & 1.0 \\
\hline Hematologic & $212(54 \%)$ & $7(41 \%)$ & $205(55 \%)$ & 0.32 \\
\hline \multicolumn{5}{|l|}{ Framingham risk score ${ }^{t}$} \\
\hline Low risk $(0 \%$ to $<4 \%)$ & $225(57 \%)$ & $2(12 \%)$ & $223(60 \%)$ & $<0.01$ \\
\hline Intermediate risk $(4 \%-14 \%)$ & $142(36 \%)$ & $8(47 \%)$ & $134(36 \%)$ & \\
\hline High risk (> 14\%) & $25(6 \%)$ & $7(41 \%)$ & $18(5 \%)$ & \\
\hline \multicolumn{5}{|l|}{ Carotid ultrasound } \\
\hline $\mathrm{IMT}(\mathrm{mm})$ & $0.65 \pm 0.12$ & $0.80 \pm 0.16$ & $0.64 \pm 0.12$ & $<0.01$ \\
\hline Presence of plaque & $127(32 \%)$ & $13(77 \%)$ & $114(30 \%)$ & $<0.01$ \\
\hline
\end{tabular}

Data are expressed as mean $\pm \mathrm{SD}$, number (percentage), or median (interquartile range).

* Diabetes mellitus was defined fasting blood glucose $>126 \mathrm{mg} / \mathrm{dl}$ and/or treatment for diabetes. Hypertension was defined as blood pressure $\geq$ 140/90 $\mathrm{mm} \mathrm{Hg}$ and/or treatment for hypertension.

${ }^{\dagger}$ Azathioprine, methotrexate, mycophenolate mofetil, mycophenolic acid, cyclophosphamide, tacrolimus, leflunomide, cyclosporine, and 6mercaptopurine.

${ }^{t}$ Redefined Framingham risk categories in terms of 8-year risk. 
政

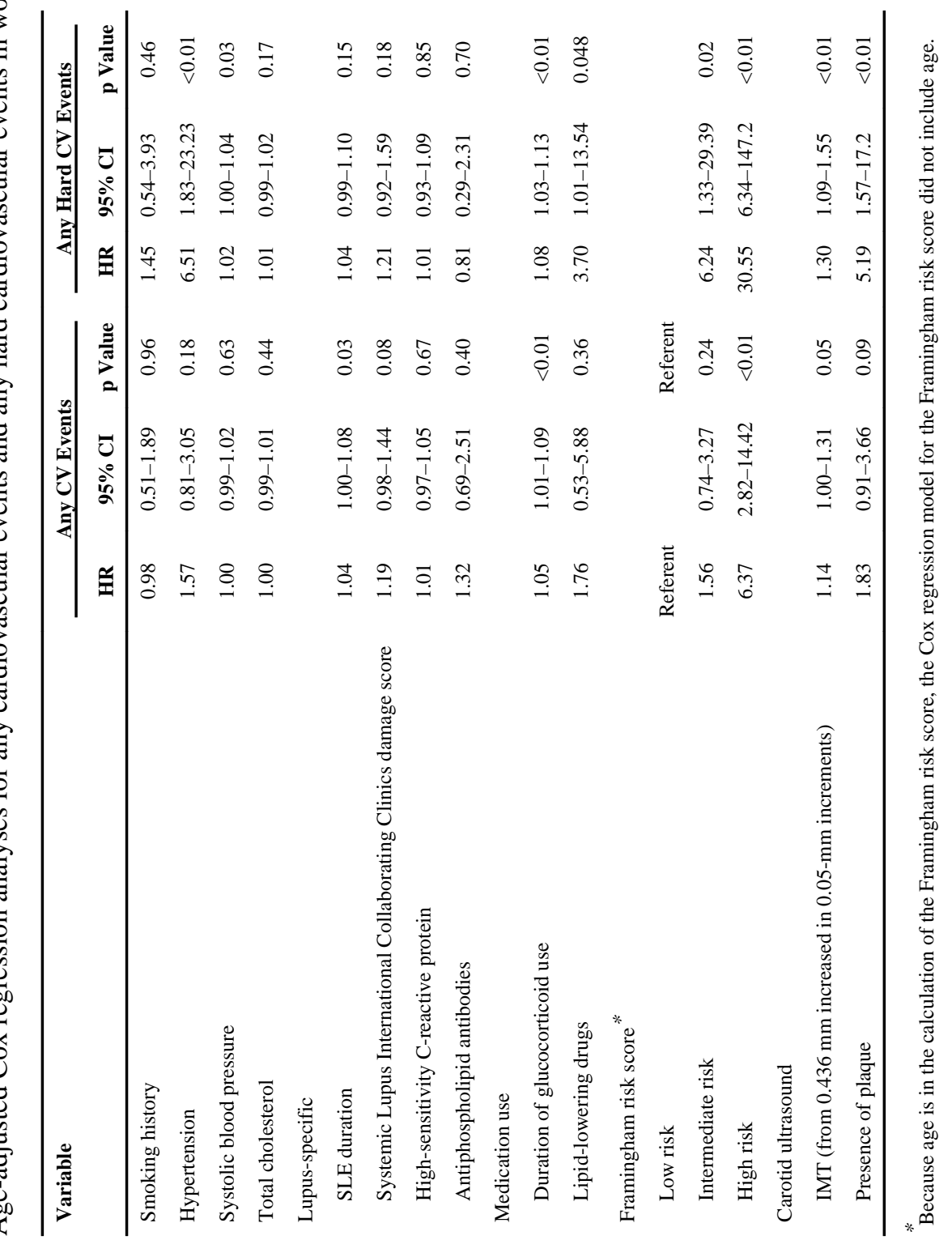




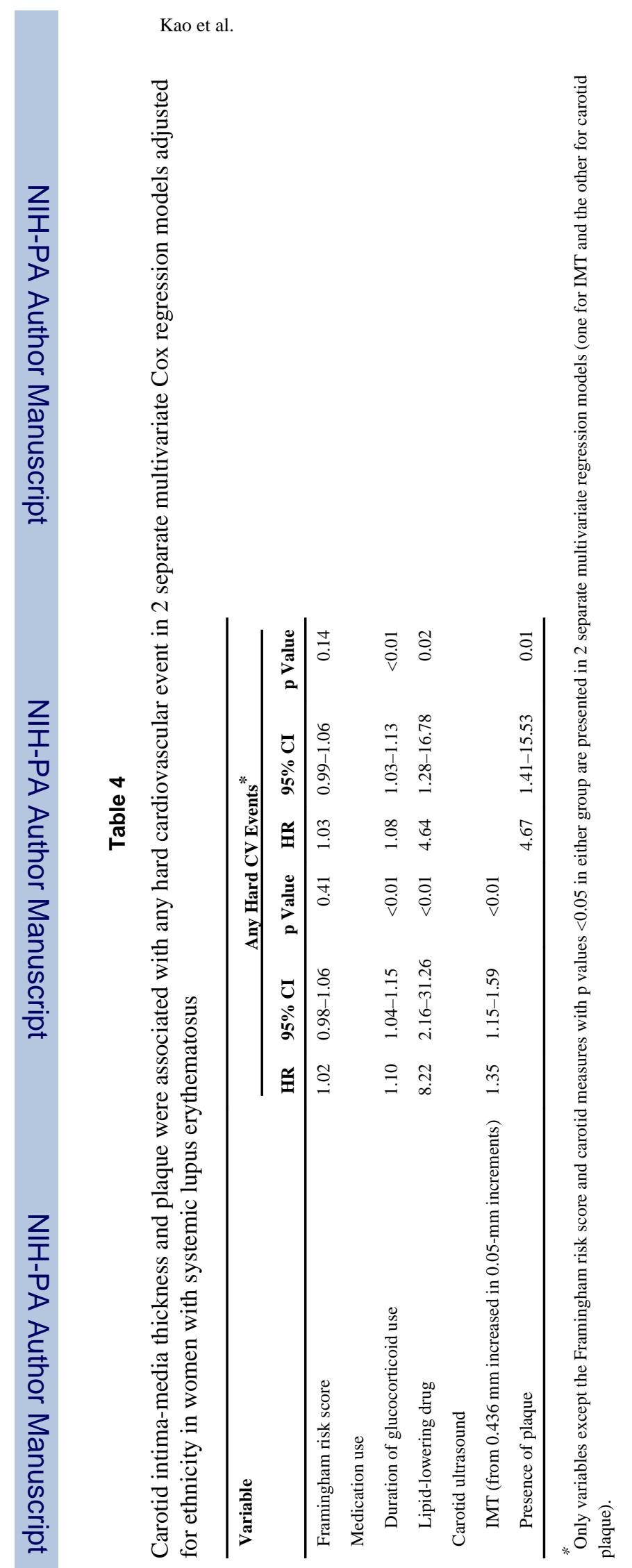

Am J Cardiol. Author manuscript; available in PMC 2014 October 01. 